\title{
Restoration of Akt activity by the bisperoxovanadium compound bpV(pic) attenuates hippocampal apoptosis in experimental neonatal pneumococcal meningitis
}

\author{
Matthias D Sury ${ }^{1}$, Lorianne Vorlet-Fawer ${ }^{1}$, Claudia Agarinis ${ }^{1}$, Shida Yousefi ${ }^{2}$, Denis \\ Grandgirard $^{1}$, Stephen L Leib ${ }^{1}$, and Stephan Christen ${ }^{1}$ \\ ${ }^{1}$ Institute of Infectious Diseases, University of Bern, CH-3010 Bern, Switzerland ${ }^{2}$ Institute of \\ Pharmacology, University of Bern, $\mathrm{CH}-3010$ Bern, Switzerland
}

\begin{abstract}
Pneumococcal meningitis causes apoptosis of developing neurons in the dentate gyrus of the hippocampus. The death of these cells is accompanied with long-term learning and memory deficits in meningitis survivors. Here, we studied the role of the PI3K/Akt (protein kinase B) survival pathway in hippocampal apoptosis in a well-characterized infant rat model of pneumococcal meningitis. Meningitis was accompanied by a significant decrease of the PI3K product phosphatidylinositol 3,4,5-triphosphate ( $\mathrm{PIP}_{3}$ ) and of phosphorylated (i.e., activated) Akt in the hippocampus. At the cellular level, phosphorylated Akt was decreased in both the granular layer and the subgranular zone of the dentate gyrus, the region where the developing neurons undergo apoptosis. Protein levels and activity of PTEN, the major antagonist of PI3K, were unaltered by infection, suggesting that the observed decrease in $\mathrm{PIP}_{3}$ and Akt phosphorylation is a result of decreased PI3K signaling. Treatment with the PTEN inhibitor bpV(pic) restored Akt activity and significantly attenuated hippocampal apoptosis. Co-treatment with the specific PI3K inhibitor LY294002 reversed restoration of Akt activity and attenuation of hippocampal apoptosis, while it had no significant effect on these parameters on its own. These results indicate that the inhibitory effect of $\mathrm{bpV}(\mathrm{pic})$ on apoptosis was mediated by PI3K-dependent activation of Akt, strongly suggesting that bpV(pic) acted on PTEN. Treatment with $\mathrm{bpV}(\mathrm{pic})$ also partially inhibited the concentration of bacteria and cytokines in the CSF, but this effect was not reversed by LY294002, indicating that the effect of bpV (pic) on apoptosis was independent of its effect on CSF bacterial burden and cytokine levels. These results indicate that the PI3K/Akt pathway plays an important role in the death and survival of developing hippocampal neurons during the acute phase of pneumococcal meningitis.
\end{abstract}

\section{Keywords}

neuronal apoptosis; inflammation; PI3K/Akt survival pathway

\footnotetext{
Address correspondence to: Prof. Stephan Christen, Institute of Infectious Diseases, Friedbuehlstrasse 51, CH-3010 Berne, Switzerland. Phone: +41 (31) 632 8707; Fax: +41 (31) 632 3550; stephan.christen@ifik.unibe.ch.

Publisher's Disclaimer: This is a PDF file of an unedited manuscript that has been accepted for publication. As a service to our customers we are providing this early version of the manuscript. The manuscript will undergo copyediting, typesetting, and review of the resulting proof before it is published in its final citable form. Please note that during the production process errors may be discovered which could affect the content, and all legal disclaimers that apply to the journal pertain.
} 


\section{Introduction}

Childhood meningitis is associated not only with a high rate of mortality but also with a high incidence of neurological long-term sequelae (Merkelbach et al., 2000; Schuchat et al., 1997). Although the incidence of bacterial meningitis has been slowly declining in developed countries since the introduction of vaccination against select pathogens, it still represents a major problem in developing countries (Tikhomirov et al., 1997). Meningitis caused by Streptococcus pneumoniae is associated with a particularly high rate of neuromotor disabilities and learning difficulties in survivors (Bedford et al., 2001). The learning difficulties (impaired memory function) have been linked to neuronal cell death in the hippocampus (Nau and Bruck, 2002). Thus, human subjects who have succumbed to pneumococcal meningitis (Nau et al., 1999) show an increased number of apoptotic neurons in the subgranular zone of the dentate gyrus. These findings are replicated in an infant rat model of pneumococcal meningitis (Pfister et al., 2000), in which neuronal damage is observed primarily in the cortex and the hippocampus. While the cortical damage is of necrotic nature, hippocampal damage occurs almost exclusively in the dentate gyrus and is of apoptotic nature, with caspase 3 being a principle mediator (Gianinazzi et al., 2003). The cells undergoing apoptosis were recently identified to be post-mitotic, developing neurons (Grandgirard et al., 2007; Sury et al., 2008). However, the upstream signaling mechanism involved in apoptotic cell death of these immature neurons is unknown.

The PI3K/Akt signaling pathway plays in an important role in the survival and cell death of neuronal cells (Brunet et al., 2001). For example, developing neurons are programmed to undergo apoptosis unless they are protected by growth factors that stimulate pro-survival pathways, among which the PI3K/Akt pathway plays a major role (Benn and Woolf, 2004). Akt (also known as protein kinase B) inhibits the activity of pro-apoptotic factors such as BH-3 only proteins (e.g., Bad) or certain transcription factors (e.g., FOXO1) (Supplementary Fig. 1) (Brunet et al., 2001; Dudek et al., 1997). Akt is activated through phosphorylation at Thr308 and Ser473 via PI3K-mediated formation of phosphatidylinositol 3,4,5-triphosphate ( $\left.\mathrm{PIP}_{3}\right)$. PI3K-induced activation of Akt is antagonized by dephosphorylation of $\mathrm{PIP}_{\mathbf{3}}$ by the lipid phosphatase PTEN (phosphatase and tensin homolog deleted on chromosome ten) (Salmena et al., 2008). Furthermore, activation of the PI3K/Akt pathway can also enhance the resistance toward extrinsic pro-apoptotic factors (Duronio, 2008; Nesterov et al., 2001).

To investigate the role of the PI3K/Akt pathway in hippocampal apoptosis caused by pneumococcal meningitis, we assessed Akt phosphorylation and nuclear translocation of its downstream target FOXO1 in the hippocampus, and analyzed the effect of the PTEN inhibitor $\mathrm{bpV}$ (pic) and the PI3K inhibitor LY294002 on hippocampal apoptosis and other clinically relevant parameters during the acute phase of pneumococcal meningitis in a well-characterized infant rat model.

\section{Materials and Methods}

\section{Infant rat model of pneumococcal meningitis}

Nursing Wistar rats, weighing 22-24 g (Charles River, Wilmington, MA), were intracisternally infected on postnatal day 11 with $10 \mu \mathrm{l}$ of saline containing $6.3 \log _{10} \mathrm{cfu} / \mathrm{ml}$ of $S$.

pneumoniae P21 or were mock-infected with $10 \mu \mathrm{l}$ of sterile saline as described previously (Gianinazzi et al., 2003; Grandgirard et al., 2007; Pfister et al., 2000). Eighteen hours post infection (p.i.), animals were clinically assessed using the following scale of scores: $1=$ coma; $2=$ does not turn upright; $3=$ turns upright within $30 \mathrm{sec} ; 4=$ minimal ambulatory activity, turns upright in less than $5 \mathrm{sec} ; 5=$ normal (Pfister et al., 2000). Subsequently, cerebrospinal fluid (CSF) was obtained by puncture of the cisterna magna and quantitatively assessed for bacterial growth. Infection and CSF sampling were performed without anesthesia. 
Myeloperoxidase (MPO) activity was analyzed in CSF pellets as an index of granulocyte infiltration as described previously (Christen et al., 2001). Antibiotic therapy was started after CSF sampling by intraperitoneal injection of ceftriaxone $(100 \mathrm{mg} / \mathrm{kg}$; Roche Pharma, Reinach Switzerland). Animals were sacrificed by decapitation at the indicated times and brains processed as described below. Animal experiments were approved by the Animal Care and Experimentation Committee of the Canton of Berne (Switzerland) and strictly followed the National Institute of Health's guidelines for the performance of animal experiments.

\section{Western blot analysis}

Western blot analysis of hippocampal homogenates was performed essentially as described before (Sury et al., 2008). Rabbit polyclonal anti phospho-Akt Thr308, phospho-Akt Ser473, phospho-PTEN Ser380, Akt or PTEN were from Cell Signaling Technologies (Beverly, MA). Bands were detected on a FluorChem SP Imaging system and band intensities determined with the Alpha Ease FC software (Alpha Innotech, San Leandro, CA).

\section{Quantification of $\mathrm{PIP}_{\mathbf{3}}$ levels}

Hippocampal lipids were extracted essentially as previously described (Gray et al., 2003; van der Kaay et al., 1997). Snap-frozen tissue was powdered in liquid nitrogen, dissolved in $0.5 \mathrm{M}$ TCA and the precipitate washed twice in 5\% TCA, 1 mM EDTA. Neutral lipids were extracted twice in $\mathrm{MeOH}: \mathrm{CHCl} 3$ (2:1) and acidic lipids were extracted in $\mathrm{MeOH}: \mathrm{CHCl} 3: 12 \mathrm{M} \mathrm{HCl}$ (80:40:1). Phase splitting was initiated by the addition of $\mathrm{CHCl} 3,0.1 \mathrm{M} \mathrm{HCl}(1: 2)$, and the organic phase dried in a vacuum centrifuge. Dried lipids were diluted in $50 \mathrm{mM}$ HEPES, 150 $\mathrm{mM} \mathrm{NaCl}, 1.5 \% \mathrm{Na}$ cholate ( $\mathrm{pH} 7.4$ ), sonicated, and stored overnight at $4^{\circ} \mathrm{C}$. PIP $\mathbf{3}$ was measured by ELISA according to the manufacturer's instructions (Echelon Biosciences $\mathrm{PIP}_{\mathbf{3}}$ Mass ELISA Kit, Salt Lake City, UT). The pellet was redissolved in $6 \mathrm{M}$ guanidine $\mathrm{HCl}$ (50 mM HEPES, $\mathrm{pH}$ 7.5) for the determination of the protein concentration.

\section{PTEN activity measurement}

Hippocampal tissue was homogenized in ice-cold IP lysis buffer $(20 \mathrm{mM}$ Tris $\mathrm{HCl}, \mathrm{pH} 7.5$, $150 \mathrm{mM} \mathrm{NaCl}, 1 \mathrm{mM}$ EDTA, $1 \%$ Triton X-100, $1 \mathrm{mM}$ PMSF, 1x protease inhibitor cocktail 1, all from Sigma-Aldrich, St. Louis, USA) under argon to avoid oxidation and under nonreducing conditions to keep the enzyme in its in vivo redox state. Protein concentration was determined by a Bradford assay (Sigma-Aldrich, St. Louis, USA). Samples were immunoprecipitated with rabbit anti-PTEN antibody (Cell Signaling Technologies, Beverly, MA). After incubation with protein A-sepharose beads (50\% slurry, EZview Red Protein A Affinity Gel, Sigma-Aldrich, St. Louis, USA), the IP pellet was washed in PTEN enzyme reaction buffer (10 mM HEPES, $150 \mathrm{mM} \mathrm{NaCl}, \mathrm{pH}$ 7.2). PTEN phosphatase activity was measured by ELISA according to the manufacturer's instructions (Echelon Biosciences PTEN ELISA, Salt Lake City, UT).

\section{Inhibitor treatment}

Animals received repetitive intraperitoneal doses $(2 \mathrm{mg} / \mathrm{kg}$ ) of bisperoxovanadate bpV(pic) (Calbiochem, San Diego, CA) or vehicle (saline) every 4 hours. This dose regimen is based on previous studies in a model of cerebral ischemia (Zhang et al., 2007). Stereotaxic injection of LY294002 $(100 \mathrm{nmol})$ into the lateral ventricle of both hemispheres $4 \mathrm{~h}$ before initiation of infection was performed as previously described (Sury et al., 2008). This dose regimen is based on previous studies in cerebral ischemia models (Kamada et al., 2007).

\section{CSF cytokine measurements}

Microsphere-based multiplex assay (Milliplex ${ }^{\circledR}$, Millipore, USA) was used to measure the CSF concentrations of the following cytokines: TNF- $\alpha$, IL-6, and IL-10. A minimum of 100 
beads per analyte was measured. Calibration curves from the recombinant standards provided were calculated with Bio-Plex Manager software version 4.1 using a five parameter logistic curve fitting. When cytokine measurements were below the detection limit, an arbitrary value corresponding to five times the detection limit of undiluted sample was used for statistical analysis.

\section{Analysis of CSF leukocytes}

To measure the total number of leukocytes, CSF was diluted ten-fold in Hank's balanced salt solution with glucose and analyzed on a Sysmex blood cell counter. To differentiate leukocyte subpopulations, cells were transferred onto glass slides using a Cytospin 3 centrifuge (Thermo scientific) and stained with May-Grünwald and Giemsa solutions.

\section{Immunofluorescence staining}

Immunofluorescence staining of $6 \mu \mathrm{m}$ paraffin sections was performed as described before (Sury et al., 2008). Goat anti-doublecortin antibody was from Santa Cruz Biotechnology (Santa Cruz, CA), rabbit polyclonal anti-phospho-Akt Thr308 and anti-FOXO1 from Cell Signaling Technologies). Secondary antibodies used were Alexa Fluor 555 donkey anti-rabbit IgG and Alexa Fluor 488 donkey anti-goat IgG (Invitrogen Corporation, Carlsbad, CA). Sections were counterstained with DAPI and mounted with ProLong Gold antifade (Invitrogen Corporation, Carlsbad, CA). Images were acquired on a wide-field epifluorescence microscope (AxioImager M1, Carl Zeiss MicroImaging GmbH, Jena, Germany) or by confocal laser scanning microscopy (LSM 5 Exciter, Carl Zeiss MicroImaging GmbH, Jena, Germany).

\section{Histopathological evaluation of apoptosis}

Histopathological evaluation of hippocampal apoptosis was performed as described in detail before (Pfister et al., 2000; Sury et al., 2008). Briefly, animals were decapitated at $24 \mathrm{~h}$ p.i. and one brain hemisphere was fixed for $24 \mathrm{~h}$ in $4 \%$ phosphate-buffered paraformaldehyde. The contralateral hemisphere was used for analysis of biochemical parameters as described above. Brain hemispheres were incubated for $24 \mathrm{~h}$ in $18 \%$ sucrose at $4^{\circ} \mathrm{C}$, snap-frozen in 2methylbutane cooled down to approx. $-40^{\circ} \mathrm{C}$, and $45 \mu \mathrm{m}$ thick coronal sections cut on a Cryostat (Jung CM1800, Leica, Glattbrugg, Switzerland). Four sections of the dorsal brain region spanning the entire depth of the dentate gyrus were stained for Nissl bodies with cresyl violet, and cells with morphological features of apoptosis (i.e., condensed fragmented dark nuclei and apoptotic bodies) were counted in the upper and the lower blade of the dentate gyrus (see Figure 4B). Numbers were averaged across the four sections. Apoptotic cells were counted by an investigator blinded to the experiment.

\section{Terminal deoxynucleotidyl transferase-mediated nick end-labeling (TUNEL) and NissI double-staining}

In some experiments, hippocampal apoptosis was assessed in $6 \mu \mathrm{m}$ paraffin sections by detection of DNA fragmentation by TUNEL staining, in combination with Nissl staining as described above. TUNEL staining was performed by using a Colorimetric FragEL ${ }^{\mathrm{TM}}$ DNA Fragmentation Detection Kit (Calbiochem) according to the manufacturer's protocol. Slides were then stained with cresyl violet, dehydrated, and mounted with Entellan (Merck, Darmstadt, Germany). TUNEL-positive and morphologically apoptotic cells were counted by bright-field microscopy exactly as described above.

\section{Statistical analysis}

Student $t$ test was used for comparison between two groups and 1-way ANOVA with Bonferroni's post-hoc test for comparison between multiple groups (Prism 4.0, GraphPad Software, San Diego, CA). Data are presented as mean \pm SEM in experiments where data are 
compared relative to a control group $(=100 \%)$, while SD was used for absolute numbers (Cumming et al., 2007). A P-value $<0.05$ was considered to be statistically significant.

\section{Results \\ Clinical disease parameters at $18 \mathrm{~h}$ p.i}

Intracisternal injection of live S. pneumoniae into 11-day-old rats lead to bacterial meningitis, as evidenced by a positive bacterial titer in the $\operatorname{CSF}(8.0 \pm 0.7 \log \mathrm{cfu} / \mathrm{ml}, \mathrm{n}=31)$, a significant reduction in the clinical score $(4.1 \pm 0.8, \mathrm{n}=33$, vs. $5.0 \pm 0.0$ in mock-infected animals, $\mathrm{n}=11$, $\mathrm{P}<0.0001)$, and loss of weight $(0.1 \pm 0.7 \mathrm{~g}, \mathrm{n}=33 \mathrm{vs}$. a weight gain of $2.4 \pm 1.7 \mathrm{~g}$ in mockinfected animals, $\mathrm{n}=11, \mathrm{P}<0.0001)$. MPO activity in CSF, as an index of granulocyte infiltration, was elevated 6700 -fold in infected $(n=11)$ compared to mock-infected animals $(\mathrm{n}=6, \mathrm{P}<0.01)$. Consistent with this finding, numbers of leukocytes in the CSF increased from $0.0 \pm 0.0(\mathrm{n}=4)$ to $600 \pm 245$ cells/ $\mu \mathrm{l}(\mathrm{n}=6, \mathrm{P}<0.5)$, of which $>85 \%$ were neutrophilic granulocytes (Table 1). CSF concentrations of the pro-inflammatory cytokines TNF- $\alpha$ and IL-6, as well as the anti-inflammatory cytokine IL-10 significantly increased from $9.3 \pm 4.2$, $33.1 \pm 18.5$, and $27.1 \pm 0.0$ (n=6 each) to $216 \pm 81.3,30^{\prime} 597 \pm 13^{\prime} 200$, and $709 \pm 446 \mathrm{pg} / \mathrm{ml}$ ( $\mathrm{n}=10$ each, $\mathrm{P}<0.001$ for all comparisons), respectively.

\section{Neonatal pneumococcal meningitis is associated with a decrease of Akt phosphorylation in the dentate gyrus of the hippocampus, but also in the cortex}

To assess the potential involvement of Akt in hippocampal apoptosis, hippocampal homogenates were prepared at different time points after infection and Akt Thr308 phosphorylation was analyzed by Western blotting (Fig. 1A). Consistent with a possible role for Akt in hippocampal apoptosis, Thr308 phosphorylation was markedly decreased ( 50\% below base line) between 24 and $40 \mathrm{~h}$ p.i.. This period of time coincides with the maximal extent of hippocampal apoptosis by histomorphometry in the infant rat model of pneumococcal meningitis (Gianinazzi et al., 2003). Akt Ser473 phosphorylation was depressed similarly between 24 and $40 \mathrm{~h}$ p.i. (data not shown). To localize the loss of Akt phosphorylation in the hippocampus, sections obtained at $24 \mathrm{~h}$ p.i. were stained for phosphorylated Thr308 and doublecortin, a marker of developing, migrating neurons, the subpopulation which undergoes apoptosis in neonatal pneumococcal meningitis (Sury et al., 2008). While Akt phosphorylation in infected animals appeared to be reduced throughout the hippocampus, the loss was most prominent in the dentate gyrus (Fig. 1B). Insets show that Akt phosphorylation was reduced both in cells of the granular layer (GL) and in doublecortin-positive migrating neurons of the subgranular zone (SGZ). Akt phosphorylation at Thr308 and Ser473 was also significantly decreased at $24 \mathrm{~h}$ and $30 \mathrm{~h}$ p.i. in the cortex (data not shown).

\section{The loss of hippocampal Akt phosphorylation during pneumococcal meningitis is due to decreased PI3K activity}

To elucidate the mechanism by which Akt phosphorylation is reduced in the hippocampus, several key components of the PI3K pathway were biochemically analyzed. In line with the reduced Akt phosphorylation in the hippocampus, hippocampal $\mathrm{PIP}_{\mathbf{3}}$ levels were significantly decreased in infected compared to mock-infected animals (Fig. 2A). To elucidate whether the decrease in hippocampal $\mathrm{PIP}_{3}$ levels might have been a consequence of increased PTEN activity, PTEN total protein and Ser380 phosphorylation were analyzed by Western blotting. However, PTEN total protein and Ser380 phosphorylation (which guides stability and activity of the protein) were unaffected by infection (Fig. 2B). Moreover, PTEN lipid phosphatase activity determined by immunocomplex assay at $24 \mathrm{~h} \mathrm{p}$.i was also unchanged (Fig. 2C). Together, these results suggest that the decreased Akt phosphorylation during pneumococcal meningitis in the hippocampus is a result of reduced PI3K activity. In line with the reduced 
Akt phosphorylation in the cortex, cortical $\mathrm{PIP}_{3}$ levels were also significantly decreased (from $15.9 \pm 3.9$ to $11.0 \pm 4.7 \mathrm{pmol} / \mathrm{mg}$ protein, $\mathrm{n}=4$ each, $\mathrm{P}<0.05$ ).

\section{Decreased hippocampal Akt phosphorylation is associated with decreased Akt activity}

To address whether decreased Akt phosphorylation is associated with decreased Akt activity, we assessed nuclear translocation of the Akt down-stream target FOXO1 (Fig. 3). Phosphorylation of FOXO1 by Akt leads to its sequestration in the cytoplasm, while unphosphorylated FOXO1 translocates to the nucleus (Birkenkamp and Coffer, 2003). In line with the marked reduction of phosphorylated Akt in the dentate gyrus, pneumococcal meningitis was associated with increased nuclear translocation of FOXO1 in cells of the GL and in migrating neurons in the SGZ (see insets). No nuclear translocation was observed in any other regions of the hippocampus (not shown).

\section{bpV(pic) treatment restores hippocampal Akt activity and inhibits hippocampal apoptosis}

Having shown that apoptosis in the hippocampal dentate gyrus is associated with decreased Akt phosphorylation, we explored whether restoration of Akt activity may decrease hippocampal apoptosis. Thus, animals were systemically treated with the potent PTEN inhibitor bpV(pic) (Schmid et al., 2004). Treatment with bpV(pic) significantly inhibited the loss of Akt phosphorylation at Thr308 (Fig. 4A) and prevented nuclear translocation of FOXO1 (Fig. 3C). This restoration of Akt phosphorylation and activity was accompanied by significant inhibition of neuronal apoptosis in the dentate gyrus of the hippocampus (Fig. 4C), assessed by histomorphometric evaluation of Nissl stained sections ( $c f$. insets Fig. 4B). To corroborate these results, apoptosis was also evaluated by TUNEL assay. In infected animals, numbers of TUNEL-positive cells (see insets Fig. 4C) showed a positive correlation with conventional histomorphological evaluation of apoptosis $\left(\mathrm{n}=9, \mathrm{P}<0.001, \mathrm{R}^{2}=0.42\right.$, slope $\left.=0.26\right)$, confirming our previous results (Bifrare et al., 2003; Gianinazzi et al., 2003). Like with conventional counting of apoptotic cells, the infection-associated increase in TUNEL-positive cells was significantly inhibited by bpV(pic) treatment (Fig. 4D). For the present study, the meningitis model was optimized for survival and the occurrence of apoptosis. To this end, a mild form of bacterial meningitis was aimed for by injection of a low inoculum. This led to a virtual absence of cortical necrosis, which precluded evaluation of the effect of $\mathrm{bpV}(\mathrm{pic})$ on cortical injury.

bpV(pic) treatment had no effect on clinical score, weight loss, CSF MPO activity, CSF leukocyte numbers and the distribution of leukocyte subpopulations in the CSF, but led to a reduction of the CSF bacterial titer by $\sim 1 \log$ (Table 1). However, linear regression analysis performed on the subset of data from paraffin sections revealed that the number of apoptotic cells in infected untreated animals is independent of the concentration of bacteria in CSF at 18 h p.i., which ranged from 6 and $9 \log _{10} \mathrm{cfu} / \mathrm{ml}\left(\mathrm{n}=13, \mathrm{P}>0.33, \mathrm{R}^{2}=0.09\right)$. To further exclude that the reduction in bacterial titer was responsible for the protective effect of $\mathrm{bpV}$ (pic), animals were co-treated with the PI3K inhibitor LY294002. While co-treatment with LY294002 completely reversed the effect of bpV(pic) on Akt phosphorylation (Fig. 4A), nuclear translocation of FOXO1 (Fig. 3D) and hippocampal apoptosis (Fig. 4C and D), it had no effect on the diminished bacterial titer (Table 1). Importantly, treatment of infected animals with LY294002 in the absence of bpV(pic) had no effect on decreased Akt phosphorylation (Fig. 4A), hippocampal apoptosis (Fig. 4C and D), bacterial titers or any of the other parameters measured (Table 1). Similar to the effect on bacterial CSF concentration, the infection-induced increase in the CSF concentration of TNF- $\alpha$, IL- 6 and IL-10 was inhibited by bpV(pic) treatment, which was not reversed by concomitant treatment with LY294002, however ( $\mathrm{P}>0.05$ by 1-way ANOVA post-hoc test). Similar to the lack of effect on bacterial burden in CSF, single treatment with LY294002 had no effect on CSF cytokine levels in infected animals. 


\section{Discussion}

In the present study, we found that experimental neonatal pneumococcal meningitis is accompanied by a marked and sustained decrease of Akt phosphorylation in the dentate gyrus between 24 and $40 \mathrm{~h}$ after infection. This decrease in phosphorylation was associated with decreased activity of the Akt survival pathway, as evidenced by the nuclear translocation of the Akt down-stream target FOXO1 in the dentate gyrus. Treatment with the PTEN inhibitor $\mathrm{bpV}$ (pic) restored Akt phosphorylation and activity, and significantly attenuated hippocampal apoptosis. These effects were completely reversed by co-treatment with the PI3K-specific inhibitor LY294002. While bpV(pic) treatment also led to a reduction in the CSF concentration of bacteria and cytokines, this effect was not reversed by co-treatment with LY294002. Treatment with LY294002 on its own did not exacerbate apoptosis or the decrease in Akt phosphorylation, or affect the infection-induced changes in the various CSF parameters (i.e., bacterial titer, cytokine levels). These results indicate that the inhibitory effect of $\mathrm{bpV}$ (pic) on apoptosis was mediated by PI3K-dependent activation of Akt and independent of its effect on CSF bacterial burden and cytokine levels. A summary of the findings of this study and the proposed mechanism by which $\mathrm{bpV}$ (pic) attenuates hippocampal apoptosis is depicted in Supplementary Fig. 2.

$\mathrm{bpV}$ (pic) was originally described as a protein tyrosine phosphatase inhibitor (Posner et al., 1994), but its potency to inhibit PTEN is about 100-fold higher when compared to that for protein tyrosine phosphatases (Schmid et al., 2004). We also performed a limited number of experiments with the even more specific PTEN inhibitor VO-OH(pic) (Rosivatz et al., 2006), which essentially yielded the same results as those obtained with $\mathrm{bpV}$ (pic) (unpublished results). However, the bisperoxovanadium compound $\mathrm{bpV}$ (pic) did have some side effects in our experimental system. Vanadium compounds have been shown to have a non-specific growth inhibitory effect on bacteria including S. pneumoniae (Fukuda and Yamase, 1997). Similar to these findings, $\mathrm{bpV}$ (pic) inhibited growth of S. pneumoniae in vitro (data not shown). However, the inhibitory effect of $\mathrm{bpV}$ (pic) in our meningitis model on apoptosis cannot be attributed to its inhibitory effect on bacterial growth, as (i) the CSF concentration of bacteria did not correlate with the extent of apoptosis, and (ii) the inhibitory effect of $\mathrm{bpV}(\mathrm{pic})$ on apoptosis was reversed by co-treatment with LY294002, while that on the reduction of CSF bacteria, was not. These results also show that the inhibitory effect of bpV(pic) on CSF bacterial numbers was likely due to the non-specific effect described for other vanadium compounds and not due to enhanced activation of a PI3K-dependent host-defense mechanism, such as phagocytosis (Schabbauer et al., 2010).

Although our experiments do not show that decreased Akt activity is a direct cause of hippocampal apoptosis (since LY294002 did only have an effect when administered together with the PTEN inhibitor), we partially explored the potential mechanism by which activity of the PI3K/Akt pathway was downregulated in pneumococcal meningitis. PTEN activity and stability is regulated by phosphorylation/dephosphorylation of C-terminal Ser residues such as Ser380 (Vazquez et al., 2000). However, we did not observe a significant change in Ser380 phosphorylation or the amount of protein. To exclude the possibility that PTEN activity may have been altered by a mechanism other than changed protein phosphorylation or stability, we assessed hippocampal PTEN activity by immunocomplex assay. We did not observe a significant change in PTEN activity (i.e., no increase), which could have explained the reduction in Akt phosphorylation accompanying pneumococcal meningitis. However, we observed a significant reduction in $\mathrm{PIP}_{3}$ levels. These results suggest that the observed loss of Akt phosphorylation is due to a reduction in PI3K signaling. However, the underlying mechanism(s) that causes this reduction remains to be determined. 
Our results show that activation of the PI3K/Akt pathway by inhibition of PTEN with bpV (pic) attenuates hippocampal apoptosis caused by pneumococcal meningitis. This is in line with our previous observation that administration of brain-derived neurotrophic factor (BDNF), which also acts via PI3K/Akt (Almeida et al., 2005), inhibits hippocampal apoptosis in this model (Bifrare et al., 2005). In addition, bpV(pic) also exhibited anti-inflammatory and anti-bacterial effects.

Development of new neurons in the dentate gyrus (neurogenesis) is known to be involved in learning and memory processes (Dupret et al., 2008; Jessberger et al., 2009). Perhaps not surprisingly, acute pneumococcal meningitis in the infant rat model is accompanied with decreased learning and memory performance three weeks after antibiosis-mediated clearance of the acute infection, as assessed by the Morris water maze test (Leib et al., 2001; Loeffler et al., 2001). Even though newly born neurons are undergoing apoptosis in the acute phase of pneumococcal meningitis, neurogenesis is known to occur lifelong in the dentate gyrus. Thus, the structure is potentially well equipped for repair. However, since in children after meningitis the neurofunctional sequelae persist for decades after the disease, the hippocampal repair potential appears to be insufficient to compensate for the brain damage (Grimwood et al., 2000).

Bisperoxovanadium compounds such as $\mathrm{bpV}$ (pic) or $\mathrm{VO}(\mathrm{OPT})$ have been shown to inhibit apoptosis also in rodent models of cerebral ischemia (Li et al., 2009; Shioda et al., 2007). Interestingly, bisperoxovanadium treatment improves neurological outcome after cerebral ischemia independent of its protective effect on the primary injury, i.e., when given several days after injury (Shioda et al., 2008). This effect is apparently due to Akt-dependent enhancement of hippocampal neurogenesis. Thus, stimulation of the PI3K/Akt pathway with PTEN inhibitors may potentially open up a new therapeutic avenue for improving long-term neurological outcome also in pneumococcal meningitis.

\section{Supplementary Material}

Refer to Web version on PubMed Central for supplementary material.

\section{Acknowledgments}

We thank Corinne Siegenthaler and Kevin Oberson for excellent technical assistance in all aspects of the study, and Corinne Boss for help with Western blot analysis. This study was supported by grants from the Swiss National Science Foundation (31-116257, 31000-120725) and the National Institute of Neurological Disorders and Stroke (R01NS33997-10A). The content is solely the responsibility of the authors and does not necessarily represent the official views of the National Institute of Neurological Disorders and Stroke or the National Institutes of Health.

\section{Abbreviations}

$\begin{array}{ll}\text { bpV(pic) } & \text { bisperoxo(pyridine-2-carboxyl)oxovanadate } \\ \text { CSF } & \text { cerebrospinal fluid } \\ \text { GL } & \text { granular layer } \\ \text { MPO } & \text { myeloperoxidase } \\ \text { NGF } & \text { nerve growth factor } \\ \text { p.i } & \text { post infection } \\ \text { PIP }_{3} & \text { phosphatidylinositol 3,4,5-triphosphate } \\ \text { PTEN } & \text { phosphatase and tension homolog deleted on chromosome } 10\end{array}$


SGZ

subgranular zone

\section{References}

Almeida RD, et al. Neuroprotection by BDNF against glutamate-induced apoptotic cell death is mediated by ERK and PI3-kinase pathways. Cell Death Differ 2005;12:1329-43. [PubMed: 15905876]

Bedford $\mathrm{H}$, et al. Meningitis in infancy in England and Wales: follow up at age 5 years. BMJ 2001;323:533-6. [PubMed: 11546697]

Benn SC, Woolf CJ. Adult neuron survival strategies--slamming on the brakes. Nat Rev Neurosci 2004;5:686-700. [PubMed: 15322527]

Bifrare YD, et al. Bacterial meningitis causes two distinct forms of cellular damage in the hippocampal dentate gyrus in infant rats. Hippocampus 2003;13:481-8. [PubMed: 12836917]

Bifrare YD, et al. Brain-derived neurotrophic factor protects against multiple forms of brain injury in bacterial meningitis. J Infect Dis 2005;191:40-5. [PubMed: 15593001]

Birkenkamp KU, Coffer PJ. Regulation of cell survival and proliferation by the FOXO (Forkhead box, class O) subfamily of Forkhead transcription factors. Biochem Soc Trans 2003;31:292-7. [PubMed: 12546704]

Brunet A, et al. Transcription-dependent and -independent control of neuronal survival by the PI3K-Akt signaling pathway. Curr Opin Neurobiol 2001;11:297-305. [PubMed: 11399427]

Christen S, et al. Oxidative stress in brain during experimental bacterial meningitis: differential effects of alpha-phenyl-tert-butyl nitrone and N-acetylcysteine treatment. Free Radic Biol Med 2001;31:75462. [PubMed: 11557313]

Cumming G, et al. Error bars in experimental biology. J Cell Biol 2007;177:7-11. [PubMed: 17420288]

Dudek H, et al. Regulation of neuronal survival by the serine-threonine protein kinase Akt. Science 1997;275:661-5. [PubMed: 9005851]

Dupret D, et al. Spatial relational memory requires hippocampal adult neurogenesis. PLoS One 2008;3:e1959. [PubMed: 18509506]

Duronio V. The life of a cell: apoptosis regulation by the PI3K/PKB pathway. Biochem J 2008;415:33344. [PubMed: 18842113]

Fukuda N, Yamase T. In vitro antibacterial activity of vanadate and vanadyl compounds against Streptococcus pneumoniae. Biol Pharm Bull 1997;20:927-30. [PubMed: 9300145]

Gianinazzi C, et al. Caspase-3 mediates hippocampal apoptosis in pneumococcal meningitis. Acta Neuropathol (Berl) 2003;105:499-507. [PubMed: 12677451]

Grandgirard D, et al. Pneumococcal meningitis induces apoptosis in recently postmitotic immature neurons in the dentate gyrus of neonatal rats. Dev Neurosci 2007;29:134-42. [PubMed: 17148956]

Gray A, et al. Nonradioactive methods for the assay of phosphoinositide 3-kinases and phosphoinositide phosphatases and selective detection of signaling lipids in cell and tissue extracts. Anal Biochem 2003;313:234-45. [PubMed: 12605860]

Grimwood K, et al. Twelve year outcomes following bacterial meningitis: further evidence for persisting effects. Arch Dis Child 2000;83:111-6. [PubMed: 10906014]

Jessberger S, et al. Dentate gyrus-specific knockdown of adult neurogenesis impairs spatial and object recognition memory in adult rats. Learn Mem 2009;16:147-54. [PubMed: 19181621]

Kamada H, et al. Bad as a converging signaling molecule between survival PI3-K/Akt and death JNK in neurons after transient focal cerebral ischemia in rats. J Cereb Blood Flow Metab 2007;27:521-33. [PubMed: 16820799]

Leib SL, et al. Inhibition of matrix metalloproteinases and tumour necrosis factor alpha converting enzyme as adjuvant therapy in pneumococcal meningitis. Brain 2001;124:1734-42. [PubMed: 11522576]

Li D, et al. Involvement of the PTEN-AKT-FOXO3a pathway in neuronal apoptosis in developing rat brain after hypoxia-ischemia. J Cereb Blood Flow Metab 2009;29:1903-13. [PubMed: 19623194] 
Loeffler JM, et al. The free radical scavenger alpha-phenyl-tert-butyl nitrone aggravates hippocampal apoptosis and learning deficits in experimental pneumococcal meningitis. J Infect Dis 2001;183:247252. [PubMed: 11110643]

Merkelbach S, et al. Cognitive outcome after bacterial meningitis. Acta Neurol Scand 2000;102:118-23. [PubMed: 10949529]

Nau R, Bruck W. Neuronal injury in bacterial meningitis: mechanisms and implications for therapy. Trends Neurosci 2002;25:38-45. [PubMed: 11801337]

Nau R, et al. Apoptosis of neurons in the dentate gyrus in humans suffering from bacterial meningitis. $\mathrm{J}$ Neuropathol Exp Neurol 1999;58:265-74. [PubMed: 10197818]

Nesterov A, et al. Elevated AKT activity protects the prostate cancer cell line LNCaP from TRAILinduced apoptosis. J Biol Chem 2001;276:10767-74. [PubMed: 11278284]

Pfister LA, et al. Endothelin inhibition improves cerebral blood flow and is neuroprotective in pneumococcal meningitis. Ann Neurol 2000;47:329-35. [PubMed: 10716252]

Posner BI, et al. Peroxovanadium compounds. A new class of potent phosphotyrosine phosphatase inhibitors which are insulin mimetics. J Biol Chem 1994;269:4596-604. [PubMed: 8308031]

Rosivatz E, et al. A small molecule inhibitor for phosphatase and tensin homologue deleted on chromosome 10 (PTEN). ACS Chem Biol 2006;1:780-90. [PubMed: 17240976]

Salmena L, et al. Tenets of PTEN tumor suppression. Cell 2008;133:403-14. [PubMed: 18455982]

Schabbauer G, et al. Myeloid PTEN promotes inflammation but impairs bactericidal activities during murine pneumococcal pneumonia. J Immunol 2010;185:468-76. [PubMed: 20505137]

Schmid AC, et al. Bisperoxovanadium compounds are potent PTEN inhibitors. FEBS Lett 2004;566:358. [PubMed: 15147864]

Schuchat A, et al. Bacterial meningitis in the United States in 1995. Active Surveillance Team. N Engl J Med 1997;337:970-6. [PubMed: 9395430]

Shioda N, et al. Bis(1-oxy-2-pyridinethiolato)oxovanadium(IV) enhances neurogenesis via phosphatidylinositol 3-kinase/Akt and extracellular signal regulated kinase activation in the hippocampal subgranular zone after mouse focal cerebral ischemia. Neuroscience 2008;155:876-87. [PubMed: 18616990]

Shioda N, et al. Activation of phosphatidylinositol 3-kinase/protein kinase B pathway by a vanadyl compound mediates its neuroprotective effect in mouse brain ischemia. Neuroscience 2007;148:2219. [PubMed: 17629407]

Sury MD, et al. JNK is activated but does not mediate hippocampal neuronal apoptosis in experimental neonatal pneumococcal meningitis. Neurobiol Dis 2008;32:142-50. [PubMed: 18703144]

Tikhomirov E, et al. Meningococcal disease: public health burden and control. World Health Stat Q 1997;50:170-7. [PubMed: 9477545]

van der Kaay J, et al. A novel, rapid, and highly sensitive mass assay for phosphatidylinositol 3,4,5trisphosphate (PtdIns(3,4,5)P3) and its application to measure insulin-stimulated PtdIns $(3,4,5) \mathrm{P} 3$ production in rat skeletal muscle in vivo. J Biol Chem 1997;272:5477-81. [PubMed: 9038150]

Vazquez F, et al. Phosphorylation of the PTEN tail regulates protein stability and function. Mol Cell Biol 2000;20:5010-8. [PubMed: 10866658]

Zhang QG, et al. Critical role of PTEN in the coupling between PI3K/Akt and JNK1/2 signaling in ischemic brain injury. FEBS Lett 2007;581:495-505. [PubMed: 17239858] 
A
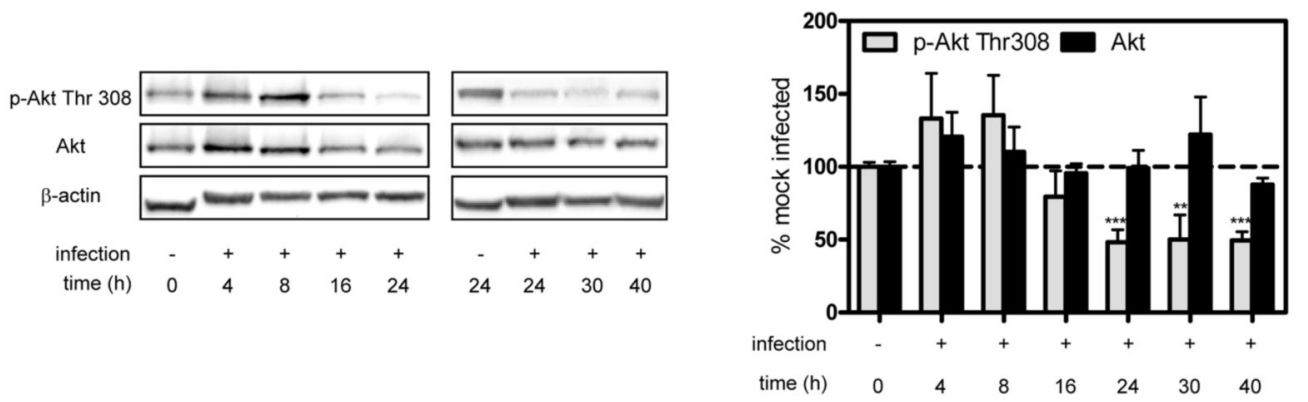

B
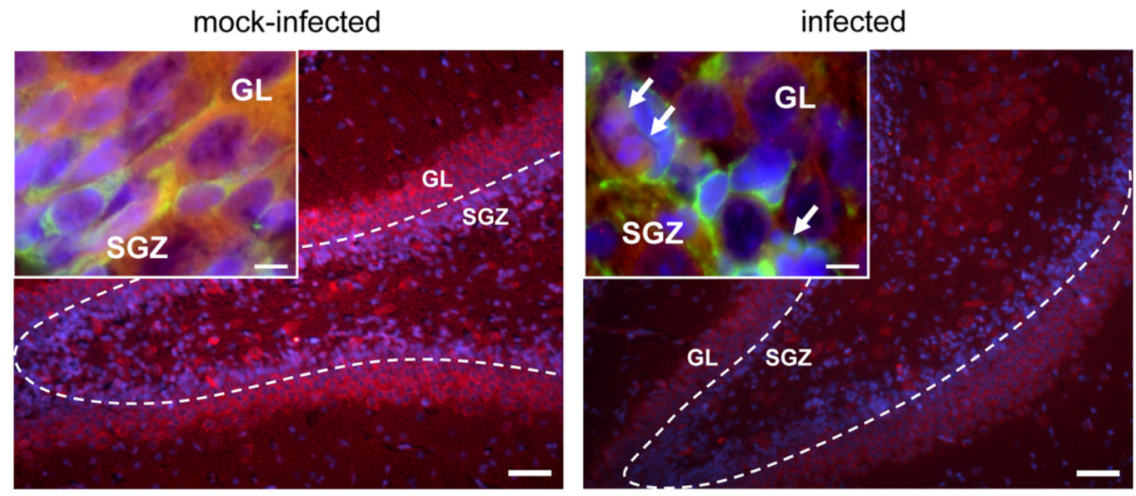

Figure 1. Kinetics and cellular localization of hippocampal Akt phosphorylation during pneumococcal meningitis

(A) Representative Western blot for phospho-Akt (Thr308) and Akt. Bar graph shows results of the densitometric analysis of the bands corrected for $\beta$-actin. Data represent the mean \pm SEM from 4-10 individual animals per time point and are expressed as the percentage relative to mock-infected animals $(=100 \%)$. Since basal levels of phospho-Akt and Akt in mock-infected animals remained unchanged at $24 \mathrm{~h}$, values were incorporated into $\mathrm{t}=0 . * * \mathrm{P}<0.01, * * * \mathrm{P}<0.001$ vs. mock-infected. (B) Staining for phospho-Akt Thr308 (red) and DAPI (blue) in the granular layer (GL) and subgranular zone (SGZ) of the hippocampal dentate gyrus. Scale bar $50 \mu \mathrm{m}$. Insets show images taken at the intersection between SGZ and GL. Doublecortin-positive cells (green) mark the end of the SGZ. Arrows point to morphologically apoptotic cells. Scale bar $10 \mu \mathrm{m}$. Images are representative of stainings obtained in sections prepared from at least 3 different animals per experimental group. 
A

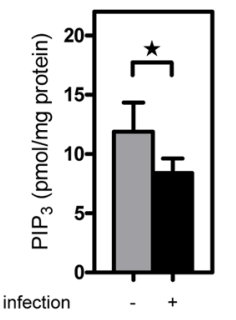

B

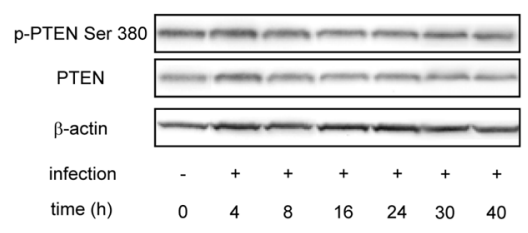

C

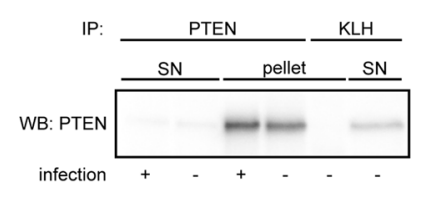

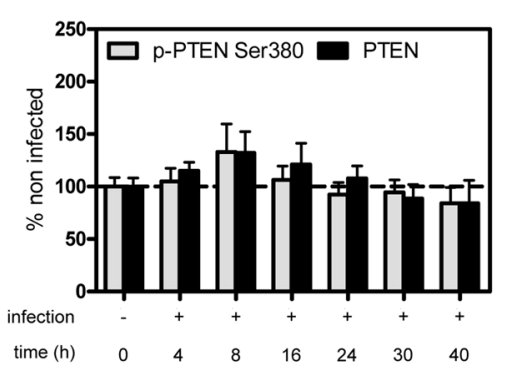

PTEN activity

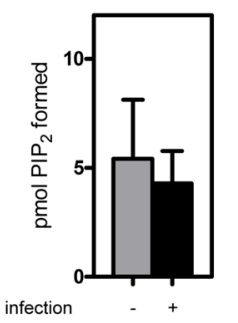

Figure 2. Effect of pneumococcal meningitis on hippocampal PIP 3 levels, PTEN phosphorylation and PTEN phosphatase activity

(A) Endogenous $\mathrm{PIP}_{3}$ levels were measured in hippocampal lipid extracts prepared at $24 \mathrm{~h}$ p.i.. Bar graph shows $\mathrm{PIP}_{3}$ levels expressed per mg protein as mean $\pm \mathrm{SD}\left(\mathrm{n}=5\right.$ each). ${ }^{*} \mathrm{P}<0.05$.

(B) Hippocampal homogenates were analyzed for PTEN Ser380 phosphorylation and total PTEN at different time points after infection by Western blot analysis. Representative blot is shown. Bar graph shows results of densitometric analysis of bands from 4-9 individual animals per time point corrected for $\beta$-actin. Data are expressed as the percentage of the mean \pm SEM of mock-infected animals $(=100 \%)$. P $>0.05$ vs. mock-infected $(t=0)$. (C) PTEN was immunoprecipitated under non-reducing/non-oxidizing conditions from hippocampal homogenates prepared at $24 \mathrm{~h}$ p.i.. An anti-key limpet hemocyanin antibody (KLH) was used as a negative control for immunoprecipitation. Representative blot is shown. PTEN activity of the immunoprecipitate was determined by measuring $\mathrm{PIP}_{\mathbf{3}}$ to $\mathrm{PIP}_{\mathbf{2}}$ conversion. Activity was adjusted for the amount of PTEN, and results are presented as mean \pm SD. No significant difference between infected $(n=8)$ and mock-infected $(n=9)$ animals. 


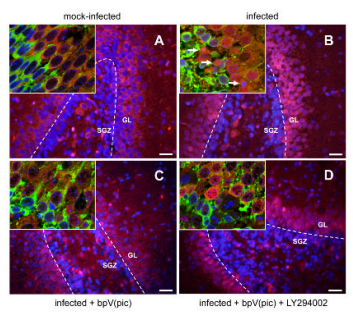

Figure 3. Effect of bpV(pic) and co-treatment with LY294002 on nuclear translocation of FOXO1 Double-staining for FOXO1 (red) and DAPI (blue) in the granular layer (GL) and subgranular zone (SGZ) of the dentate gyrus in the hippocampus. Scale bar $50 \mu \mathrm{m}$. Insets show confocal laser scanning microscopy images taken at the intersection between SGZ and GL.

Doublecortin-positive cells (green) mark the end of the SGZ. Arrows point to morphologically apoptotic cells. Images are representative of stainings obtained in sections prepared from at least 3 different animals per experimental group. 


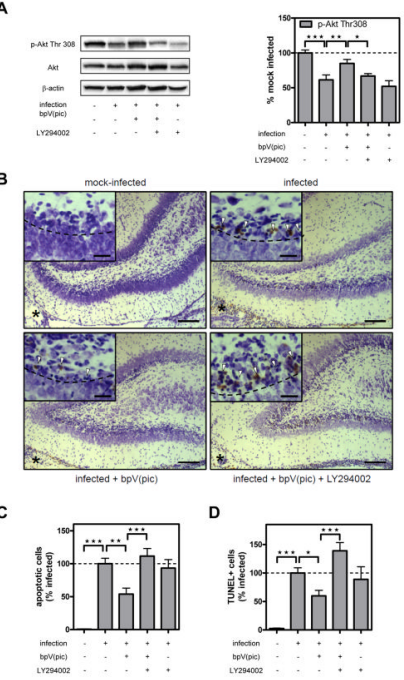

Figure 4. Effect of bpV(pic) and co-treatment with LY294002 on Akt Thr308 phosphorylation and hippocampal apoptosis

(A) Representative phospho-Akt (Thr308) and Akt Western blot. Total Akt levels were unaffected by any of the inhibitor treatments. Bar graph shows results of densitometric analysis of phospho-Akt band from 6-10 individual animals per time point corrected for $\beta$-actin. Data are expressed as the percentage of the mean \pm SEM of mock-infected animals $(=100 \%)$. $* \mathrm{P}<0.05, * * \mathrm{P}<0.01$ vs. mock-infected. (B) Images show representative Nissl and TUNEL double-stained sections of the dentate gyrus of animals mock-infected (top left), infected (top right), infected $+\mathrm{bpV}$ (pic) (bottom left) or infected + bpV(pic) + LY294002 (bottom right). Asterisk marks ventricle adjacent to the dentate gyrus of the hippocampus, which are inflamed in infected animals. Scale bar $50 \mu \mathrm{m}$. Arrowheads in the insets point at cells with apoptotic morphology (i.e., presence of apoptotic bodies or fragmented dark nuclei) and/or TUNELpositive cells. Scale bar $20 \mu \mathrm{m}$. (C) Morphologically apoptotic cells were counted in the upper and lower blade of the dentate gyrus, and the mean was calculated from four sections spanning the entire hippocampus. Results represent the mean \pm SEM number of apoptotic cells from $\mathrm{n}=6$ (mock-infected), 17 (infected), 11 (infected $+\mathrm{bpV}($ pic $), 16$ (infected $+\mathrm{bpV}($ pic $)+$ LY294002), and 15 (infected + LY294002) animals expressed relative to infected animals without treatment $(=100 \%)$. $* * \mathrm{P}<0.01$ and $* * * \mathrm{P}<0.001$. Stereotaxic treatment with DMSO alone had no effect on meningitis-induced apoptosis (not shown). (D) TUNEL-positive cells were counted in the upper and lower blade of the dentate gyrus, and the mean was calculated from four sections spanning the entire hippocampus. Results represent the mean \pm SEM number of TUNEL-positive cells from $n=3$ (mock-infected), 9 (infected), 8 (infected $+\mathrm{bpV}$ (pic), 7 (infected + bpV(pic) + LY294002), and 7 (infected + LY294002) animals expressed relative to infected animals without treatment $(=100 \%)$. $* \mathrm{P}<0.05$ and $* * * \mathrm{P}<0.001$. 


\section{Table 1}

Effect of bpV(pic) and/or LY294002 treatment on clinical disease and inflammatory parameters in infected animals.

\begin{tabular}{|c|c|c|c|c|}
\hline Treatment & vehicle & bpV(pic) & bpV(pic) + LY294002 & LY294002 \\
\hline CSF bacterial titer $\left(\log _{10} \mathrm{cfu} / \mathrm{ml}\right)$ & $7.9 \pm 0.8(13)$ & $* * 6.9 \pm 0.7(16)$ & $* * 6.9 \pm 0.9(12)$ & $8.0 \pm 0.7(10)$ \\
\hline$\Delta$ weight (g) (mock-inf - inf) & $2.4 \pm 0.7(17)$ & $2.0 \pm 1.1(16)$ & $2.5 \pm 0.9(15)$ & $2.4 \pm 1.4(11)$ \\
\hline Clinical score (1-5) & $3.9 \pm 0.2(17)$ & $3.9 \pm 0.3(16)$ & $3.9 \pm 0.4(15)$ & $4.0 \pm 0.0(11)$ \\
\hline CSF MPO activity (\% infected) & $100 \pm 23.4(7)$ & $110 \pm 27.0(6)$ & $78.6 \pm 13.6(10)$ & $113 \pm 22.1(9)$ \\
\hline CSF leukocytes (per $\mu \mathrm{l}$ ) & $600 \pm 245(6)$ & $625 \pm 386(4)$ & $500 \pm 743(4)$ & $480 \pm 164(5)$ \\
\hline$\%$ Neutrophils & $84.5 \pm 9.2(7)$ & $92.6 \pm 4.8(7)$ & $92.9 \pm 6.5(7)$ & $90.0 \pm 3.9(6)$ \\
\hline \%Monocytes & $10.9 \pm 4.7(7)$ & $5.7 \pm 4.8(7)$ & $4.4 \pm 5.5(7)$ & $7.2 \pm 3.3(6)$ \\
\hline \%Lymphocytes & $4.5 \pm 5.0(7)$ & $1.7 \pm 1.0(7)$ & $2.7 \pm 2.6(7)$ & $2.6 \pm 1.4(6)$ \\
\hline \%Eosinophils & $0.1 \pm 0.2(7)$ & $0.0 \pm 0.0(7)$ & $0.0 \pm 0.0(7)$ & $0.2 \pm 0.3(6)$ \\
\hline CSF TNF- $\alpha(\%$ infected) & $100 \pm 11.9(10)$ & $* 41.3 \pm 6.2(11)$ & $* * 32.8 \pm 9.6(8)$ & $126 \pm 24.5(8)$ \\
\hline CSF IL-6 (\%infected) & $100 \pm 13.6(10)$ & $* * * 29.6 \pm 6.3(11)$ & $* 49.4 \pm 9.4(8)$ & $96.4 \pm 17.8(8)$ \\
\hline CSF IL-10 (\% infected) & $100 \pm 19.9(10)$ & $* * * 22.1 \pm 5.6(11)$ & $* * 32.8 \pm 9.6(8)$ & $72.7 \pm 11.4(8)$ \\
\hline
\end{tabular}

All parameters were determined at $18 \mathrm{~h}$ p.i. Results are given as mean $\pm \mathrm{SD}$ for absolute data and mean $\pm \mathrm{SEM}$ for data expressed relative to infected, vehicle-treated animals (\%infected) with number of animals in brackets.

$* *$

$P<0.01$ and

$* * *$

$P<0.001$ vs. infected, vehicle-treated animals.

LY294002 co-treatment did not reverse the reduction in CSF bacterial titer and cytokine concentrations mediated by bpV(pic) treatment (P $>0.05,1-$ way ANOVA post-hoc test). 